\title{
Efficient simulation and equilibrium theory for adsorption processes with implicit adsorption isotherms - Mass action equilibria
}

\author{
M. Fechtner ${ }^{\mathrm{a}}$, A. Kienle $\mathbf{e}^{\mathrm{a}, \mathrm{b}, *}$ \\ ${ }^{a}$ Otto-von-Guericke-Universität, Universitätsplatz 2, D-39106 Magdeburg, \\ Germany \\ ${ }^{b}$ Max-Planck-Institut für Dynamik komplexer technischer Systeme, \\ Sandtorstrasse 1, D-39106 Magdeburg, Germany
}

\begin{abstract}
An efficient method for the simulation of packed bed adsorbers is presented. It is based on equilibrium models and can be applied to any implicit adsorption isotherm. It uses a method of lines approach, avoids explicit differentiation of the adsorption isotherm, and exploits standard numerics for the simultaneous solution of the resulting ordinary differential and implicit algebraic equations. Application is illustrated for stoichiometric ion exchange, which also admits an analytical approach using equilibrium theory. Classical theoretical results from equilibrium theory are summarized, further extended and compared to the numerical calculations for different scenarios. Focus is on selectivity reversals and their influence on process operation. It is shown that the selectivity reversal may introduce multiple solutions.
\end{abstract}

Keywords: Adsorption, Simulation, Differential algebraic systems, Stoichiometric ion exchange, Equilibrium theory

\footnotetext{
*Author to whom all correspondence should be addressed.

Email address: kienle@mpi-magdeburg.mpg.de (A. Kienle)
} 


\section{Introduction}

Mathematical modeling of spatially distributed packed bed adsorbers leads to systems of partial differential equations. An important class of models assume thermodynamic equilibrium between the fluid and the solid phase [1, 2]. Most

5 important representatives of this class are the equilibrium dispersive model with axial dispersion and the ideal equilibrium model, without axial dispersion. The equilibrium dispersive model is often used for a more quantitative prediction of adsorber dynamics. The dispersion lumps together all effects causing band broadening. An analytical solution is only possible for special cases, i.e. for 10 linear isotherms or the calculation of single shock profiles [2, 3]. Therefore, usually a numerical solution is applied.

In contrast to this, the ideal model without axial dispersion provides more qualitative insight, but admits an analytical approach using the method of characteristics and builds the basis of the so called equilibrium theory. Equilibrium

15 theory is a powerful approach to analyze and understand the dynamic behavior of adsorption processes and is therefore also an important tool for process design [4]. Classical equilibrium theory is for Langmuir isotherms [2] or ion exchange with constant separation factors [5]. More recently, extensions were given to Bi-Langmuir [6], 7], generalized Langmuir [8], generalized Bi-Langmuir [9], and also to reactive systems with simultaneous phase and reaction equilibrium [10, 11, 12].

Besides explicit adsorption isotherms also implicit formulations are quite common to describe the adsorption equilibrium. Typical examples are stoichiometric ion exchange [13, 14, 5] and the ideal adsorbed (IAS) and real adsorbed

25 solution (RAS) theory approaches [15]. These are computationally much more difficult to treat due to the implicit phase equilibrium. Usually some challenging implicit analytical or numerical differentiation of the equilibrium relations is required to calculate the capacity matrix of the model equations [16, 17]. In the present paper we propose a much easier alternative approach. It uses method of lines [18] and a reformulation of the underlying adsorber model equations in the 
form of a differential algebraic equation (DAE) system with differential index 1

[19]. It can be solved with available standard software for DAEs and thereby avoids explicit differentiation of the adsorption isotherm.

In this paper, application is demonstrated for stoichiometric ion exchange

35 with mass action equilibria. This is a classical problem, which has been treated first by Tondeur et al. 13, 14, 5]. A characteristic feature are possible selectivity reversals predicted by this model. It also admits an analytical approach. In the present paper, results are further extended to provide a full picture of possible transitions.

40 The outline of the paper is as follows: First the numerical approach is introduced. Afterwards, a rigorous analysis is given based on equilibrium theory. Focus is on selectivity reversals and their impact on operability and uniqueness of solutions. Theoretical findings are validated with numerical simulations.

\section{Modeling and simulation}

45 The following is based on the well known equilibrium dispersive model of a packed bed adsorber. It assumes isothermal operation, thermodynamic equilibrium between the adsorbed and the fluid phase, constant interstitial velocity $v$ of the fluid phase and a constant void fraction $\epsilon$. In dimensionless form the model reads

$$
\frac{\partial}{\partial t}(\mathbf{c}+F \mathbf{q}(\mathbf{c}))+\frac{\partial \mathbf{c}}{\partial z}=\frac{1}{P e} \frac{\partial^{2} \mathbf{c}}{\partial z^{2}} \quad \text { with } \quad \mathbf{c}, \mathbf{q} \in R^{N}
$$

with dimensionless time and space coordinates

$$
z=z^{*} / L, \quad t=t^{*} v / L
$$

and dimensionless quantities

$$
F=(1-\epsilon) / \epsilon, \quad P e=v L / D
$$
sponding to $1 / P e \rightarrow 0$. 
In this model equations $\mathbf{c}$ represents the concentrations of the $N$ adsorbable components in the fluid phase, and $\mathbf{q}(\mathbf{c})$ the corresponding concentrations in the adsorbed phase, which follow from the adsorption equilibrium. Focus in the 55 present paper is on implicit adsorption equilibria

$$
\mathbf{0}=\mathbf{f}(\mathbf{q}, \mathbf{c})
$$

where $\mathbf{q}$ is an implicit function of $\mathbf{c}$. Typical examples are stoichiometric ion exchange with mass action equilibrium (see e.g. [5]) or the ideal or real adsorbed solution theory (see e.g. [15]). In the remainder, stoichiometric ion exchange will be considered as a first application example. However, the solution strategy

6o can also be applied to the other implicit adsorption equilibria.

In stoichiometric ion exchange, typically, a constant solution normality $c_{t o t}=$ $\sum_{i=1}^{N} \mu_{i} c_{i}$ and a fixed exchanger capacity $q_{t o t}=\sum_{i=1}^{N} \mu_{i} q_{i}$ can be assumed. Therein, the $\mu$ variables represent the valences of the ionic species. This reduces the degrees of freedom of Eq. (11) by one and normalized concentrations $x_{i}=$ ${ }_{65} \mu_{i} c_{i} / c_{t o t}, y_{i}=\mu_{i} q_{i} / q_{t o t}$ can be introduced, with $\sum_{i=1}^{N} x_{i}=1, \sum_{i=1}^{N} y_{i}=1$. In normalized concentrations the model equations without physical dispersion read

$$
\frac{\partial}{\partial t}(\mathbf{x}+\kappa \mathbf{y}(\mathbf{x}))+\frac{\partial \mathbf{x}}{\partial z}=0
$$

with $\kappa=F q_{t o t} / c_{t o t}$. Due to the above summation conditions for the $\mathbf{x}$ and $\mathbf{y}$ variables only $N-1$ equations of (3) are required. The equilibrium relations have to be reformulated accordingly. For stoichiometric ion exchange, for example, we find

$$
K_{i N}=\left(\frac{y_{i}}{x_{i}}\right)^{\nu_{i}}\left(\frac{x_{N}}{y_{N}}\right)^{\nu_{N}}=\text { const. }, \quad i=1, \ldots, N-1 .
$$

for any reference component ' $N$ '. Following the notation in [5], the $\nu$ variables are the reciprocal valences of the different ionic species. It is worth noting that this notation is slightly different from [20, 13, 14] who used valences instead of reciprocals in their formulation. 
${ }_{75} \quad$ For equal $\nu_{i}=\nu_{N}=\nu$ for all $i=1, \ldots, N-1$ we obtain constant separation factors $\alpha_{i N}$ according to

$$
\alpha_{i N}=\left(\frac{y_{i}}{x_{i}}\right)\left(\frac{x_{N}}{y_{N}}\right)=K_{i N}^{\frac{1}{\nu}}=\text { const. }
$$

and Eqs. (4) can be solved explicitly for

$$
y_{i}=\frac{\alpha_{i N} x_{i}}{1+\sum_{k=1}^{N-1}\left(\alpha_{k N}-1\right) x_{k}} .
$$

If the valences of the different species are not equal, the separation factors are no longer constant and Eqs. (4) represent a set of $N-1$ implicit algebraic

so equations to calculate the $y_{i}$ 's from the $x_{i}$ 's. These equations can be put to the form

$$
0=f_{i}(\mathbf{y}, \mathbf{x})=1-\frac{1}{K_{i N}} \cdot\left(\frac{y_{i}}{x_{i}}\right)^{\nu_{i}}\left(\frac{x_{N}}{y_{N}}\right)^{\nu_{N}}, \quad \forall i=1, \ldots, N-1
$$

Alternatively, we may reformulate Eqs. (4) as a single implicit equation and a set of $N-1$ explicit equations [20]. However, in view of applicability to other implicit adsorption equilibria we will use formulation (7) in the remainder.

85 For an efficient numerical solution of the model equations (3) for the packed bed adsorber we introduce the quantity

$$
\mathbf{w}=\mathbf{x}+\kappa \mathbf{y}(\mathbf{x})
$$

which reflects the joint capacity of the fluid and the adsorbed phase in Eq. (3). Note, that $\mathbf{w}$ could be normalized by division with $1+\kappa$, which however has no effect on the general procedure and is therefore not considered here. In so terms of $\mathbf{w}$ and $\mathbf{x}$ the model equations can now be reformulated as

$$
\begin{gathered}
\frac{\partial \mathbf{w}}{\partial t}+\frac{\partial \mathbf{x}}{\partial z}=\mathbf{0}, \\
\mathbf{0}=\mathbf{f}\left(\mathbf{x}, \mathbf{y}=\frac{\mathbf{w}-\mathbf{x}}{\kappa}\right),
\end{gathered}
$$


which are solved simultaneously for $\mathbf{w}$ and $\mathbf{x}$ using method of lines [18]. For a proof of principle, in the present paper, a simple discretization of the spatial coordinate using first order backward differences on an equidistant grid is applied. However, application of our simulation strategy to more economic

95 discretization formulas using for example high resolution methods [21] is straight forward. In all of these cases, method of lines leads to a system of differential equations and implicit algebraic equations (DAEs) with differential index one [19], which can be solved with standard software like DASSL [22] or LIMEX 23] or ODE15s in Matlab 24]. For a discussion of the differential index we refer to Appendix A.

One of the main challenges for the solution of DAE systems is the calculation of consistent starting conditions. In the present case, focus is on so called Riemann problems with piecewise constant initial and boundary conditions. For this case consistent initial conditions are easily calculated with an offline evaluation of the adsorption equilibrium for given fluid phase composition $\mathbf{x}$ in the feed and given initial condition $\mathbf{y}$ of the bed.

Main advantage of our new solution strategy is that we avoid explicit differentiation of the adsorption isotherm in formulation (9), (10) compared to the standard formulations (1) or (2). Differentiation of adsorption isotherms 110 is especially difficult in the case of implicit adsorption isotherms considered in this paper. Further, the new method can also be useful for complicated explicit adsorption isotherms if the explicit differentiation is challenging.

\section{Equilibrium theory}

Equation (3) represents a system of quasilinear partial differential equations 115 of first order, which can also be solved (semi-) analytically for piecewise constant initial and boundary conditions using the method of characteristics. For this purpose Eq. (3) is rewritten as

$$
\frac{\partial \mathbf{x}}{\partial t}+\left(\mathbf{I}_{N-1}+\kappa \frac{\partial \mathbf{y}}{\partial \mathbf{x}}\right)^{-1} \frac{\partial \mathbf{x}}{\partial z}=0
$$


where $\mathbf{I}_{N-1}$ is the $(N-1) \times(N-1)$ identity matrix. In contrast to previous work [20, 13, 14, 5] so called 'adjusted' times and velocities are omitted in this model formulation for the clarity of presentation.

Solutions of (3) or (11) with Riemann boundary and initial conditions consist of smooth transitions - also called simple, spreading or dispersive waves, and shock or self sharpening waves [2, 25]. Further, we will show that any selectivity reversal with

$$
\frac{y_{k}}{x_{k}}=\frac{y_{k+1}}{x_{k+1}}
$$

is associated with a contact discontinuity which is linearly degenerate and therefore neither spreading nor self sharpening [2, 25].

Any concentration of a smooth transition is traveling with characteristic velocity $\sigma_{k}$ corresponding to the eigenvalues of matrix $\left(\mathbf{I}_{N-1}+\kappa \frac{\partial \mathbf{y}}{\partial \mathbf{x}}\right)^{-1}$ in Eq. (11)

$$
\sigma_{k}=\frac{1}{1+\kappa \lambda_{k}}
$$

where the $\lambda$ 's are the eigenvalues of $\frac{\partial \mathbf{y}}{\partial \mathbf{x}}$.

From implicit differentiation of Eq. (7) we obtain

$$
\frac{\partial \mathbf{y}}{\partial \mathbf{x}}=-\left(\frac{\partial \mathbf{f}}{\partial \mathbf{y}}\right)^{-1} \frac{\partial \mathbf{f}}{\partial \mathbf{x}}
$$

with

$$
\begin{aligned}
-\frac{\partial \mathbf{f}}{\partial \mathbf{y}} & =\operatorname{diag}_{N-1}\left(\frac{\nu_{i}}{y_{i}}\right)+\mathbf{1}_{N-1} \frac{\nu_{N}}{y_{N}} \\
\frac{\partial \mathbf{f}}{\partial \mathbf{x}} & =\operatorname{diag}_{N-1}\left(\frac{\nu_{i}}{x_{i}}\right)+\mathbf{1}_{N-1} \frac{\nu_{N}}{x_{N}}
\end{aligned}
$$

where $\operatorname{diag}_{N-1}\left(z_{i}\right)$ stands for the $(N-1) \times(N-1)$ diagonal matrix with elements $z_{i}, \quad \forall i=1, \ldots, N-1$ and $\mathbf{1}_{N-1}$ is a $(N-1) \times(N-1)$ matrix where all entries are equal to one. With this, the characteristic equation for the calculation of the eigenvalues $\lambda_{k}$ can be written as 


$$
\begin{aligned}
0 & =\operatorname{det}\left(\frac{\partial \mathbf{y}}{\partial \mathbf{x}}-\lambda_{k} \mathbf{I}_{N-1}\right) \\
& =\operatorname{det}\left(-\frac{\partial \mathbf{f}}{\partial \mathbf{x}}-\lambda_{k} \frac{\partial \mathbf{f}}{\partial \mathbf{y}}\right) \\
& =\operatorname{det}\left(\operatorname{diag}_{N-1}\left(\frac{\nu_{i}}{x_{i}}-\lambda_{k} \frac{\nu_{i}}{y_{i}}\right)+\mathbf{1}_{N-1}\left(\frac{\nu_{N}}{x_{N}}-\lambda_{k} \frac{\nu_{N}}{y_{N}}\right)\right)
\end{aligned}
$$

For $\lambda_{k} \neq \frac{y_{i}}{x_{i}}$ the characteristic equation can be expanded into

$$
0=\sum_{i=1}^{N} \frac{1}{\frac{\nu_{i}}{x_{i}}-\lambda_{k} \frac{\nu_{i}}{y_{i}}}
$$

which has $N-1$ real and distinct roots in the intervals

$$
\frac{y_{1}}{x_{1}}>\lambda_{1}>\frac{y_{2}}{x_{2}}>\ldots>\frac{y_{k}}{x_{k}}>\lambda_{k}>\frac{y_{k+1}}{x_{k+1}}>\ldots>\frac{y_{N-1}}{x_{N-1}}>\lambda_{N-1}>\frac{y_{N}}{x_{N}} .
$$

If the components are ordered in decreasing selectivity for the solid phase.

For $\lambda_{k}=\frac{y_{i}}{x_{i}}$ we find from Eq. (19) $\lambda_{k}=\frac{y_{i}}{x_{i}}=\frac{y_{N}}{x_{N}}$ for any reference component ' $N$ '. If the above ordering of components is applied, this is only possible between neighboring components undergoing a selectivity reversal with indices $i, N$ being equal to $k, k+1$. In view of Eq. (4) we find that the characteristic velocity is constant along the selectivity reversal according to

$$
\lambda_{k}=\frac{y_{k}}{x_{k}}=\frac{y_{k+1}}{x_{k+1}}=K_{k, k+1}^{\frac{1}{\nu_{k}-\nu_{k+1}}}
$$

giving rise to a contact discontinuity.

The image of the smooth transitions in the concentration or hodograph space is given by the corresponding eigenvectors $\mathbf{v}_{k}$. For $\lambda_{k} \neq \frac{y_{k}}{x_{k}}$ the eigenvectors follow from

$$
0=\left(\frac{\nu_{i}}{x_{i}}-\lambda_{k} \frac{\nu_{i}}{y_{i}}\right) v_{k, i}+\left(\frac{\nu_{N}}{x_{N}}-\lambda_{k} \frac{\nu_{N}}{y_{N}}\right) \sum_{m=1}^{N-1} v_{k, m}, \quad \forall i=1, \ldots, N-1
$$


In view of the characteristic equation (20) an obvious choice to satisfy this

150 equation is

$$
\mathbf{v}_{k}=\left[\begin{array}{cc}
\frac{1}{\frac{\nu_{1}}{x_{1}}-\lambda_{k} \frac{\nu_{1}}{y_{1}}}, \ldots & \frac{1}{\frac{\nu_{N-1}}{x_{N-1}}-\lambda_{k} \frac{\nu_{N-1}}{y_{N-1}}}
\end{array}\right]^{T} .
$$

For the selectivity reversal with $\lambda_{k}=\frac{y_{k}}{x_{k}}$ we find

$$
\mathbf{v}_{k}=\left[0, \ldots 0, v_{k, k}=1, v_{k, k+1}=-1,0, \ldots 0\right]^{T}, \quad \forall k=1, \ldots, N-1
$$

corresponding to a straight line along which all concentrations are constant except for components ' $k$ ' and ' $k+1$ '. In case of a ternary system, the two possible selectivity reversal lines defined by $[1,-1]$ and $[0,1]$ are parallel to the $155 x_{3}=1-x_{1}-x_{2}=0$-line and the $x_{2}$-axis, respectively.

Using the above expressions for $\lambda_{k}$ and $\mathbf{v}_{k}$, it can be proven that the characteristic velocity along the $\mathrm{k}$-th characteristic is changing monotonically for $\lambda_{k} \neq \frac{y_{k}}{x_{k}}$ according to

$$
\nabla \lambda_{k} \mathbf{v}_{k} \neq 0
$$

Similar results were reported by [26] for stoichiometric ion exchange without selectivity reversals.

For spreading waves the characteristic velocity is monotonically increasing in the direction of increasing $z$, whereas for shock waves the characteristic velocity is monotonically decreasing in the direction of increasing $z$. The shock velocity $s^{k}$ follows from the integral material balances across the shock also known as

$$
s^{k}=\frac{1}{1+\kappa \frac{\Delta y_{i}}{\Delta x_{i}}}, \forall i=1, \ldots, N-1 .
$$

These equations also define the image of the shock waves in the hodograph space, similar to the eigenvectors introduced above. In general, eigenvectors and shock curves are tangent to each other [25]. For $\nu_{i}=\nu_{N}$ for all $i=1, \ldots, N-1$, 
corresponding to the case of constant separation factors, both types of curves are straight lines and coincide. For $\nu_{i} \neq \nu_{N}$ they are curved and therefore different, but still close in the cases to be discussed subsequently, so that the following qualitative discussion will be based on the path grid of the eigenvectors only. However, existence of the different wave solutions was also checked on a rigorous basis using entropy conditions [25].

175 4. Results

A ternary example, with one selectivity reversal indicated by the dashed line is shown in Fig. 1. Corresponding parameters are given in Table 1. In region I component 1 is stronger adsorbed, whereas in region II component 2 is stronger adsorbed. The red curves were calculated from the eigenvector $v_{1}$ corresponding to the eigenvalue $\lambda_{1}$, which satisfies $\lambda_{1}>\lambda_{2}$. Since the characteristic speed in equation (13) depends on the reciprocal of the $\lambda$ 's, the eigenvector $v_{1}$ represents the family of slow waves. The arrows are pointing in the direction of increasing characteristic velocity. The orientation of the red curves is reverted from region I to region II, whereas the orientation of the blue curves is uniform in the whole composition space. The characteristic velocity along the selectivity reversal is constant as discussed in the previous section. Point $\mathrm{W}$ represents a watershed point where the eigenvalues coincide. According to the above discussion this can only happen on the boundary of the concentration triangle.

In the remainder different characteristic scenarios are discussed. For all loading and regeneration scenarios, the numerical plateau values in the profiles are identical with equilibrium theory solutions in the hodograph. Wave profiles depend on the number of grid points but show very similar behavior as predicted by the equilibrium theory. First focus is on the loading of an empty bed with a feed in region I as shown in Fig. 2. Initial and feed composition are represented by points $\mathrm{F}$ and $\mathrm{I}$ in Fig. 2a. The solution consists of a shock wave $s_{1}$ corresponding to the path $\mathrm{F}_{-} \mathrm{P}_{1}$ in Fig. $2 \mathrm{a}$ and a shock wave $s_{2}$ corresponding to the path $\mathrm{P}_{1}$-I in Fig. 2a. Corresponding simulation results using backward 
differences with 750 equidistant grid points are shown in Fig. 2b. The behavior shown in Fig. 2 is similar to a system with constant separation factors or a Langmuirian system with component 1 being the stronger adsorbed component. It shows the characteristic intermediate plateau for the weaker adsorbed component with increased composition compared to the adjacent plateaus of the feed and the initial conditions.

The situation is reverted for a loading of an empty bed with feed in region II 205 as illustrated in Fig. 3. Here, the behavior is similar to a system with constant separation factors or a Langmuirian system with component 2 being the stronger adsorbed component and is therefore some sort of mirror image of the situation in Fig. 2.

The limiting case with a feed composition on the selectivity reversal is shown in Fig. 4. Here, the shock velocities for the shocks $\mathrm{P}_{1}$-I and $\mathrm{P}_{2}$-I along the $x_{1}$ and the $x_{2}$ axis coincide with the constant characteristic velocity along the selectivity reversal. Hence, both solutions $F-P_{1}-I$ and $F-P_{2}-I$ are feasible. It can be further shown, that any other point on the selectivity reversal is connected to the origin by a shock wave with the same velocity like the shocks $\mathrm{P}_{1}$-I, and ${ }_{215} \mathrm{P}_{2}$-I leading to a whole spectrum of possible solutions. Similar phenomena were reported in [27, 28] for distillation processes. Which one of this solutions is obtained in simulation depends on the intrinsic stability of the different wave solutions and on numerical dispersion, which is introduced by discretization. A simulation example with 200 and 2000 grid points is shown in Fig. 5. A rigorous mathematical stability analysis of the underlying partial differential equations with dispersion is challenging and clearly beyond the scope of this work.

From the practical point of view, Fig. 4 represents a singular situation which nicely explains the transition between the two different patterns in Figs. 2 and 3 but can not be observed as such in practice due to fluctuations. Classical existence and uniqueness theorems in the mathematical literature are usually confined to local situations where points $\mathrm{F}$ and I are sufficiently close (see e.g. [25], Theorem 17.18) and therefore do not apply here.

Next focus is on chromatographic cycles, which were also not addressed in 
the classical literature [13, 14, 5]. The chromatographic cycle corresponds to a pulse disturbance, consisting of the loading of an empty bed in the front followed by the regeneration of the loaded bed in the rear.

A first scenario with a feed in region I is shown in Figure 6a. The loading in the front corresponds to Fig. 2. It consists of two shocks, with a band of pure component 2 in the front, which is the weaker adsorbed component in region I. The regeneration consists two spreading waves $r_{1}$ and $r_{2}$ illustrated in yellow in Fig. 6a. Due to the special topology of the path grid $r_{1}$ coincides with the $x_{2}$ axis generating also a band of pure component 2 in the rear. Corresponding simulation results for the regeneration are shown in Fig. 6b. Together with the corresponding loading profiles in Fig $2 \mathrm{~b}$, the complete cycle can be reconstructed. After interaction of the different fronts the pattern will be resolved in two pure component pulses with pure component 1 in the front and pure component 2 in the rear as shown in Fig. 7. This is due to the fact that during wave interactions the selectivity reversal is crossed and the final resolution is taking place in region II, where component 1 is the weaker adsorbed component.

This is also confirmed with a second scenario with feed in region II, which is illustrated in Figure 8. Loading in the front corresponds to the previous Fig. 3 consisting of two shock waves with a band of pure component 1 in the front which is the weaker adsorbing component in region II. Regeneration in the rear consists of two spreading waves $r_{1}$ and $r_{2}$ illustrated in yellow in Fig. 8a. Again, simulation results for the regeneration that are shown in Fig. 8b can be used to obtain the complete cycle with the loading profiles of Fig. 3b. The topology of the hodograph in II is simpler than in I and more similar to a Langmuirian system, leading to a band of pure component 2 in the rear. After elementary interaction of the different fronts the pattern will be resolved 255 in two pure component pulses, as shown in Fig. 9, with pure component 1 in the front and pure component 2 in the rear like in the previous case. This clearly shows that the influence of the selectivity reversal strongly depends on the mode of operation. For the step inputs in Figs 2 and 3 qualitatively different final patterns were obtained, whereas for the pulse inputs only the transients 
were different but those were then resolved in similar final patterns.

Like in Fig. 4 multiple solutions are also possible for the chromatographic cycle if the feed composition is located on the selectivity reversal as shown in Fig. 10. Following the argumentation above both solution will be resolved in the same final pattern.

Additional features can occur in systems with multiple selectivity reversals as illustrated with an example in Fig. 11. Parameters are given in Table 2. Like in the previous case, a selectivity reversal between components 1 and 2 is observed at the boundary between regions I and II. In addition, a selectivity reversal between components 2 and 3 occurs at the boundary between regions I and III. An interesting feature which was also reported by [13, 14] is the occurrence of wave patterns with more than $N-1$ wave fronts as illustrated in Fig. 11. This theoretical prediction could also be validated with the new numerical approach presented in this paper as illustrated in Fig. 11b. For better resolution 1000 equidistant grid points were used in Fig. 11b.

Finally, it should be mentioned that this finding is also consistent with theoretical results. In theory it is stated that the solution of a Riemann problem of a strictly hyperbolic system, genuinely nonlinear or linearly degenerate system consists of a maximum number of $N-1$ wave transitions (see [25], Theorem 17.18). However, again the Theorem is confined to local situations, where points F and I are sufficiently close (see e.g. [25]).

\section{Conclusions}

An efficient method for the numerical solution of equilibrium models of fixed bed adsorbers with implicit adsorption isotherms was presented. As a first application example, stoichiometric ion exchange with possible selectivity reversals was considered. The effect of selectivity reversals on process operation was studied systematically using a combined numerical, analytical approach. Besides the validation of the new numerical approach also interesting patterns of behavior were found complementing previous studies for this particular kind of system. 
Future work will be concerned with an application of the new approach to more 290 challenging systems described by the IAS or RAS theory.

\section{Acknowledgments.}

The financial support of the International Max Planck Research School for Advanced Methods in Process and Systems Engineering - IMPRS ProEng is greatly acknowledged. 
Notation

\begin{tabular}{|c|c|c|}
\hline $\mathbf{1}_{N}$ & matrix of ones size $N \times N$ & {$[-]$} \\
\hline$\alpha_{i N}$ & separation factor of component $\mathrm{i}$ and reference component $\mathrm{N}$ & {$[-]$} \\
\hline$c_{i}$ & fluid phase concentration of component i & {$\left[\frac{m o l}{l}\right]$} \\
\hline$c_{t o t}$ & solution normality & {$\left[\frac{\mathrm{mol}}{l}\right]$} \\
\hline$D$ & dispersion & {$\left[\frac{m^{2}}{s}\right]$} \\
\hline$\epsilon$ & void fraction & {$[-]$} \\
\hline$F$ & phase ratio & {$[-]$} \\
\hline $\mathbf{I}_{N}$ & identity matrix size $N \times N$ & {$[-]$} \\
\hline$K_{i N}$ & equilibrium constant of component $\mathrm{i}$ and reference component $\mathrm{N}$ & {$[-]$} \\
\hline$\kappa$ & adjusted phase ratio & {$[-]$} \\
\hline$L$ & length of column & {$[m]$} \\
\hline$\lambda_{k}$ & $\mathrm{k}$-th eigenvalue & {$[-]$} \\
\hline$\mu_{i}$ & valence of component $\mathrm{i}$ & {$[-]$} \\
\hline$N$ & number of components & {$[-]$} \\
\hline$N_{z}$ & number of grid points & {$[-]$} \\
\hline$\nu_{i}$ & stoichiometric coefficient of component i & {$[-]$} \\
\hline$P e$ & Péclet number & {$[-]$} \\
\hline$q_{i}$ & solid phase concentration of component i & {$\left[\frac{m o l}{l}\right]$} \\
\hline$q_{\text {tot }}$ & exchanger capacity & {$\left[\frac{m o l}{l}\right]$} \\
\hline$v_{k, i}$ & i-th entry of k-th eigenvector & {$[-]$} \\
\hline$r_{k}$ & $\mathrm{k}$-th spreading wave & {$[-]$} \\
\hline$s^{k}$ & k-th shock velocity & {$[-]$} \\
\hline$s_{k}$ & k-th shock & {$[-]$} \\
\hline$\sigma_{k}$ & k-th characteristic velocity & {$[-]$} \\
\hline$t$ & dimensionless time coordinate & {$[-]$} \\
\hline$t^{*}$ & time coordinate & {$[s]$} \\
\hline$v$ & interstitial velocity & {$\left[\frac{m}{s}\right]$} \\
\hline$w_{i}$ & conserved flux variable of component i & {$[-]$} \\
\hline$x_{i}$ & normalized fluid phase concentration of component i & {$[-]$} \\
\hline$y_{i}$ & normalized solid phase concentration of component i & {$[-]$} \\
\hline$z$ & dimensionless space coordinat 55 & {$[-]$} \\
\hline$z^{*}$ & space coordinate & {$[m]$} \\
\hline
\end{tabular}




\section{References}

[1] H.-K. Rhee, R. Aris, N. R. Amundson, First-Order Partial Differential Equations: Volume I - Theory and Application of Single Equations, Prentice Hall, New Jersey, 1986.

[2] H.-K. Rhee, R. Aris, N. R. Amundson, First-Order Partial Differential Equations: Volume II - Theory and Application of Hyperbolic Systems of Quasilinear Equations, Prentice Hall, New Jersey, 1989.

[3] M. Mazzotti, G. Storti, M. Morbidelli, Shock layer analysis in multicomponent chromatography and countercurrent adsorption, Chemical Engineering Science 49 (1994) 1337-1355.

[4] M. Mazzotti, A. Rajendran, Equilibrium theory-based analysis of nonlinear waves in separation processes, Annual Review of Chemical and Biomolecular Engineering 4 (2013) 119-141.

[5] F. G. Helfferich, G. Klein, Multicomponent Chromatography. Theory of Interference, M. Dekker, New York, 1970.

[6] A. Butté, G. Storti, M. Mazzotti, Shock formation in binary systems with nonlinear characteristic curves, Chemical Engineering Science 63 (2008) 4159-4170.

[7] D. Flockerzi, M. Kaspereit, A. Kienle, Spectral properties of Bi-Langmuir isotherms, Chemical Engineering Science 104 (2013) 957-959.

[8] M. Mazzotti, Local equilibrium theory for the binary chromatography of species subject to a generalized Langmuir isotherm, Industrial \& Engineering Chemistry Research 45 (2006) 5332-5350.

[9] F. Ortner, S. Jermann, L. Joss, M. Mazzotti, Equilibrium theory analysis of a binary system subject to a mixed generalized Bi-Langmuir isotherm, Industrial \& Engineering Chemistry Research 54 (2015) 11420-11437. 
[10] S. Grüner, A. Kienle, Equilibrium theory and nonlinear waves for reactive distillation columns and chromatographic reactors, Chemical Engineering Science 59 (2004) 901-918.

[11] T. D. Vu, A. Seidel-Morgenstern, S. Grüner, A. Kienle, Analysis of ester hydrolysis reactions in a chromatographic reactor using equilibrium theory and a rate model, Industrial \& Engineering Chemistry Research 44 (2005) 9565-9574.

[12] S. Grüner, M. Mangold, A. Kienle, Dynamics of reaction separation processes in the limit of chemical equilibirium, AIChE Journal 52 (2006) 10101026.

[13] D. Tondeur, Theorie des colonnes d'change d'ions, ph.D. Thesis, Universite de Nancy (1969).

[14] D. Tondeur, Theory of ion-exchnage columns, Chemical Engineering Journal 1 (1970) 337-346.

[15] D. M. Ruthven, Principles of Adsorption and Adsorption Processes, John Wiley \& Sons, 1984.

[16] K. Kaczmarski, D. Antos, Calculation of chromatographic band profiles with an implicit isotherm, Journal of Chromatography A 862 (1999) 1-16.

[17] H. O. R. Landa, D. Flockerzi, A. Seidel-Morgenstern, A method for efficiently solving the IAST equations with aqn application to adsorber dynamics, AIChE Journal 59 (2013) 1263-1277.

[18] W. E. Schiesser, The Numerical Method of Lines Integration of Partial Differential Equations, Academic Press, San Diego, 1991.

345 [19] J. Unger, A. Kröner, W. Marquardt, Structural analysis of differentialalgebraic equation systems: Theory and applications, Computers \& Chemical Engineering 19 (1995) 867-882. 
[20] G. Klein, D. Tondeur, T. Vermeulen, Multicomponent ion exchange in fixed beds, Industrial \& Engineering Chemistry Fundamentals 6 (1967) 339-351.

[21] R. J. LeVeque, Numerical Methods for Conservation Laws, Birkhäuser Verlag, Basel, 1992.

[22] K. E. Brenan, S. L. Campbell, L. R. Petzold, Numerical Solution of Initial Value Problems in Differential-Algebraic Equations, North Holland \& Elsevier Science Publishing Company, 1989.

355 [23] P. Deuflhard, E. Hairer, J. Zugck, One-step and extrapolation methods for differential-algebraic systems, Numerische Mathematik 51 (1987) 501-516.

[24] MATLAB, version 8.4.0 (R2014b), The MathWorks Inc., Natick, Massachusetts, 2014.

[25] J. Smoller, Shock Waves and Reaction-Diffusion Equations, 2nd Edition, Springer, New York, 1994.

[26] H. P. Urbach, On the conservation laws of nonlinear chromatography for heterovalent ions, Proceedings of the Royal Society A 440 (1993) 303-322.

[27] A. Kienle, W. Marquardt, Bifurcation analysis and steady-state multiplicity of multicomponent, non-equilibrium distillation processes, Chemical Engineering Science 46 (1991) 1757-1769.

[28] F. G. Helfferich, Multiple steady states in multicomponent countercurrent mass-transfer processes, Chemical Engineering Science 48 (1993) 681-686.

[29] E. Kvaalen, L. Neel, D. Tondeur, Directions of quasi-static mass and energy transfer between phases in multicomponent open systems, Chemical Engineering Science 40 (1985) 1191-1204.

\section{Appendix A. Differential Index of the DAE System}

In the remainder, it is shown that the PDAE system (9), (10), or the corresponding DAE system resulting from the discretization of (9), (10) using a 
method of lines approach, respectively, has differential index 1 , whenever the Jacobian $\partial \mathbf{y} / \partial \mathbf{x}$ has $N-1$ real, positive eigenvalues $\lambda_{i}$. For thermodynamic reasons this should always be the case [29]. Explicit proofs for Langmuir isotherms were given in [29], for Bi-Langmuir isotherms in [7] and for the IAS theory for a large class of pure component isotherms in [17], Appendix B. Hence, the result of this Appendix does not only apply to mass action equilibria but is valid for any thermodynamically reasonable sorption equilibrium.

The PDAE system (91), (10) has differential index 1 when the matrix of the derivatives of the algebraic equations (10) with respect to the algebraic variable $\mathrm{x}$ is nonsingular. From differentiation of Eq. (10) we find

$$
\left(\frac{\partial \mathbf{f}}{\partial \mathbf{x}}\right)_{\mathbf{w}}=\left(\frac{\partial \mathbf{f}}{\partial \mathbf{x}}\right)_{\mathbf{y}}-\frac{1}{\kappa}\left(\frac{\partial \mathbf{f}}{\partial \mathbf{y}}\right)_{\mathbf{x}}
$$

Therein, the indices at the brackets indicate which variable is constant during differentiation.

For the calculation of the Jacobian $\partial \mathbf{y} / \partial \mathbf{x}$, the equilibrium composition of the solid phase $\mathbf{y}$ is interpreted as a function of the fluid phase composition $\mathbf{x}$. From implicit differentiation of the equilibrium relation

$$
\mathbf{0}=\mathbf{f}(\mathbf{x}, \mathbf{y}(\mathbf{x}))
$$

we find

$$
\frac{\partial \mathbf{y}}{\partial \mathbf{x}}=-\left(\frac{\partial \mathbf{f}}{\partial \mathbf{y}}\right)_{\mathbf{x}}^{-1}\left(\frac{\partial \mathbf{f}}{\partial \mathbf{x}}\right)_{\mathbf{y}}
$$

Since $\partial \mathbf{y} / \partial \mathbf{x}$ has $N-1$ positive nonzero eigenvalues $\lambda_{i}$, it is regular, i.e. its determinant is nonzero. Further, due to the product rule for determinants both matrices on the right hand side of the above equation also have to be regular. Substitution of Eq. (A.3) into Eq. (A.1) yields

$$
\left(\frac{\partial \mathbf{f}}{\partial \mathbf{x}}\right)_{\mathbf{w}}=-\left(\frac{\partial \mathbf{f}}{\partial \mathbf{y}}\right)_{\mathbf{x}}\left(\frac{\partial \mathbf{y}}{\partial \mathbf{x}}+\frac{1}{\kappa} \mathbf{I}\right)
$$

where $\mathbf{I}$ is the $(N-1) \times(N-1)$ identity. The eigenvalues of 


$$
\left(\frac{\partial \mathbf{y}}{\partial \mathbf{x}}+\frac{1}{\kappa} \mathbf{I}\right)
$$

are equal to $\lambda_{i}+1 / \kappa$ and are therefore also nonzero and the corresponding matrix is regular. Hence, the matrix on the left hand side of Eq. (A.4) is a product of two regular matrices and therefore also regular, which completes the proof.

Finally, the DAE system resulting from the discretization of (9), (10) using 400 a method of lines approach has also differential index 1, if the above is satisfied at any spatial position in the reactor. This however, is trivial since the above results are valid for any concentrations. 
List of Tables

1 Parameters of the example with one selectivity reversal. . . . . 22

$405 \quad 2 \quad$ Parameters of the example with two selectivity reversals. . . . . . 23 


\begin{tabular}{|c|c|c|}
\hline parameter & value & description \\
\hline$L$ & 15.0 & column length \\
\hline$N_{z}$ & 750 & number of grid points \\
\hline$v$ & 1.0 & interstitial velocity \\
\hline$\varepsilon$ & 0.5 & void fraction \\
\hline$c_{t o t}$ & 0.18 & solution normality \\
\hline$q_{t o t}$ & 2.0 & exchanger capacity \\
\hline$K_{13}$ & 4.0 & equilibrium constant \\
\hline$K_{23}$ & 2.67 & equilibrium constant \\
\hline$\nu_{1}$ & 2 & stoichiometric factor \\
\hline$\nu_{2}$ & 1 & stoichiometric factor \\
\hline$\nu_{3}$ & 1 & stoichiometric factor \\
\hline
\end{tabular}

Table 1: Parameters of the first example system with one selectivity reversal. 


\begin{tabular}{|c|c|c|}
\hline parameter & value & description \\
\hline$L$ & 20.0 & column length \\
\hline$N_{z}$ & 1000 & number of grid points \\
\hline$v$ & 1.0 & interstitial velocity \\
\hline$\varepsilon$ & 0.5 & void fraction \\
\hline$c_{t o t}$ & 0.01 & solution normality \\
\hline$q_{t o t}$ & 2.0 & exchanger capacity \\
\hline$K_{13}$ & 100.0 & equilibrium constant \\
\hline$K_{23}$ & 14.88 & equilibrium constant \\
\hline$\nu_{1}$ & 4 & stoichiometric factor \\
\hline$\nu_{2}$ & 1 & stoichiometric factor \\
\hline$\nu_{3}$ & 4 & stoichiometric factor \\
\hline
\end{tabular}

Table 2: Parameters of the second example system with two selectivity reversals. 


\section{List of Figures}

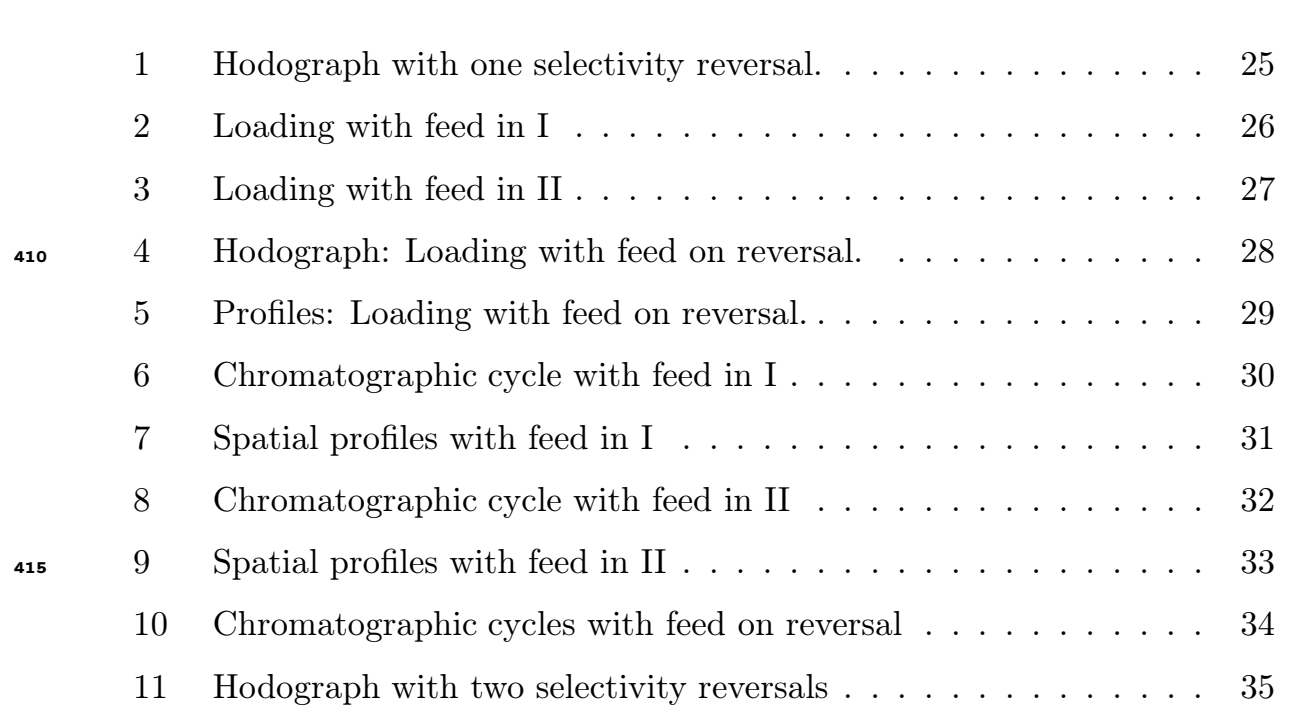




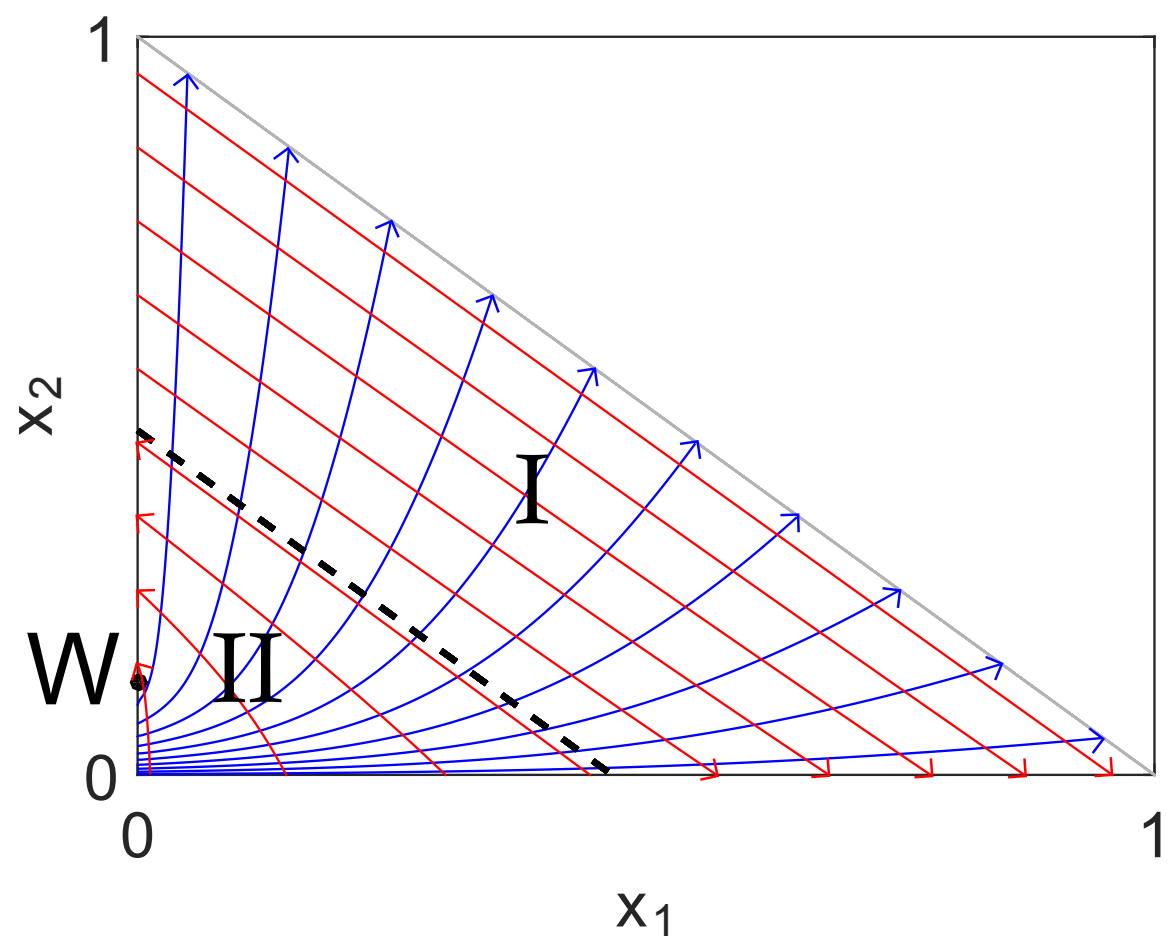

Figure 1: Hodograph of the first example system with one selectivity reversal. 
(a)

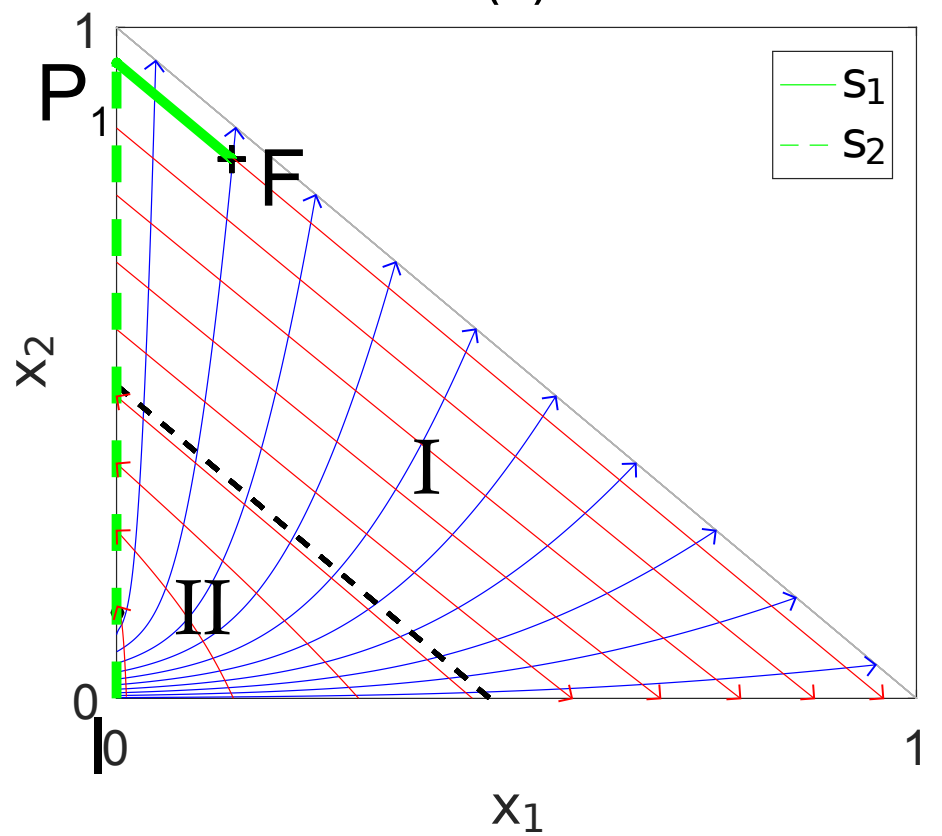

(b)

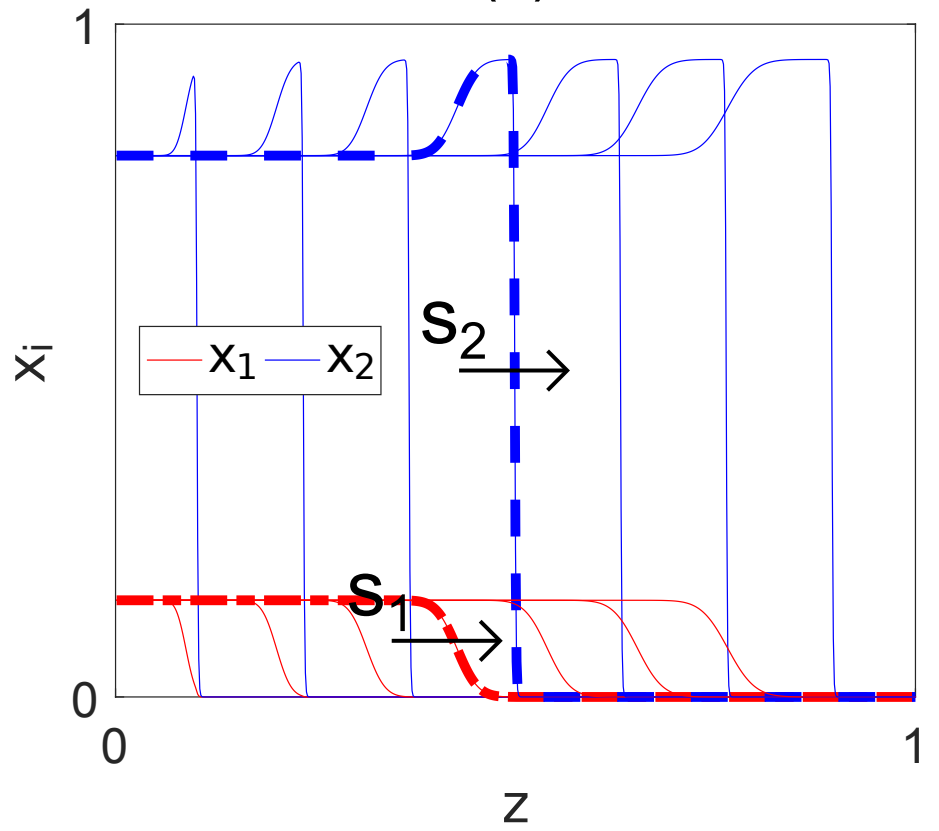

26

Figure 2: (a) Solution in the hodograph space for the loading of an empty bed with $F=$ $[0.1437,0.8046]^{T}$ in I. (b) Corresponding spatial profiles $x_{i}(z)$ at different time points with. 
(a)

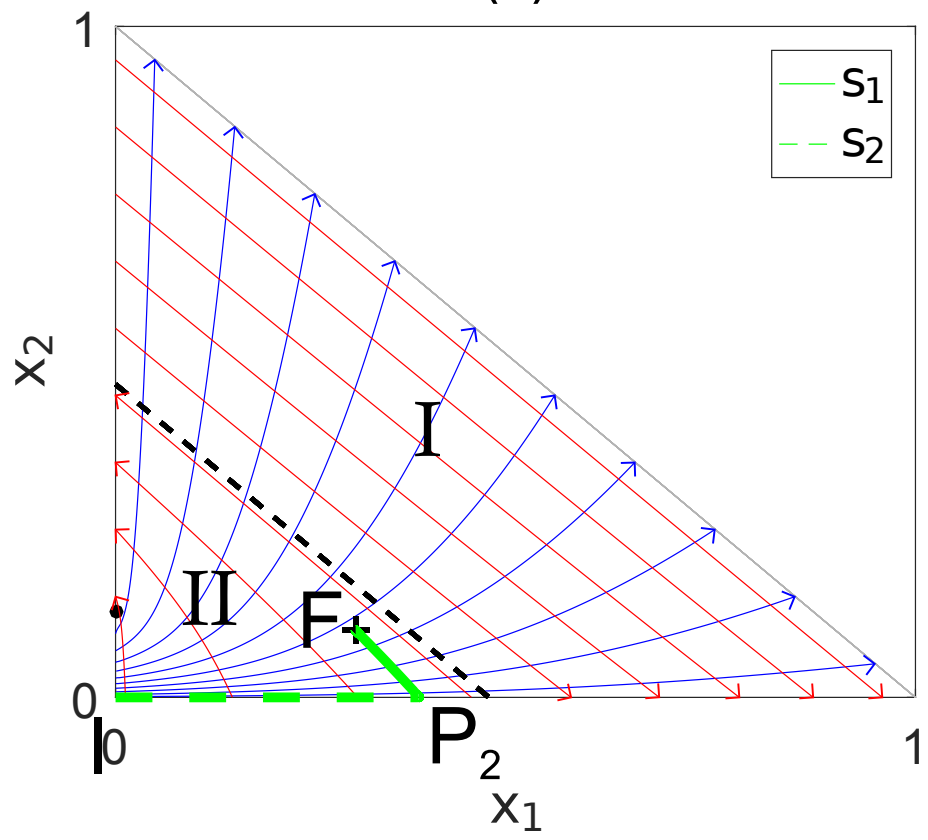

(b)

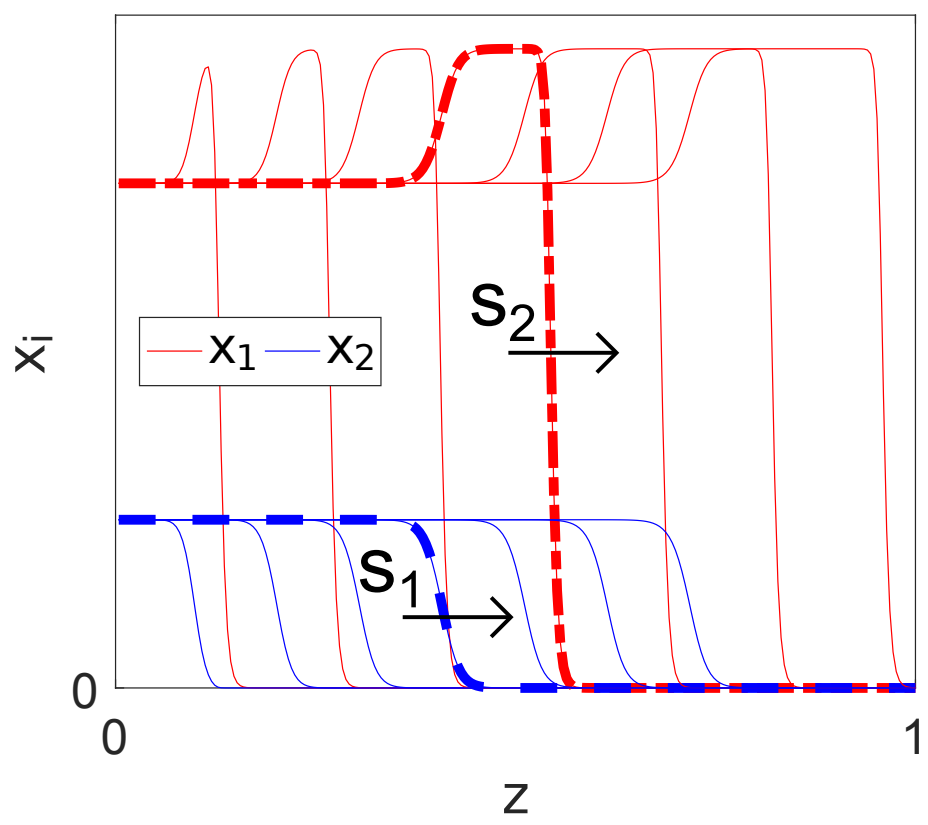

27

Figure 3: (a) Solution in the hodograph space for the loading of an empty bed with $F=$ $[0.3,0.1]^{T}$ in II. (b) Corresponding spatial profiles $x_{i}(z)$ at different time points. 


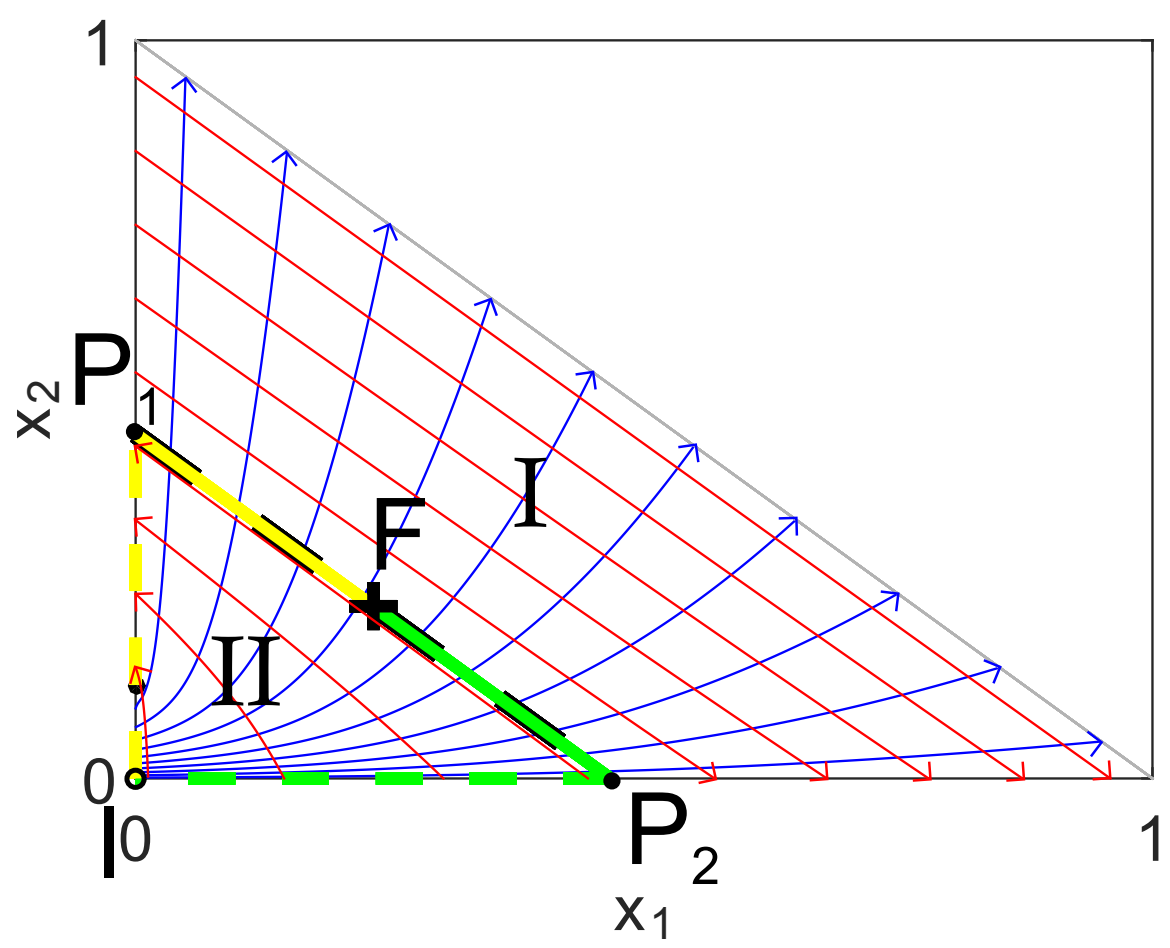

Figure 4: Two possible solutions in the hodograph space for the loading of an empty bed with $F=[7 / 30,7 / 30]^{T}$ on the selectivity reversal. 
(a)

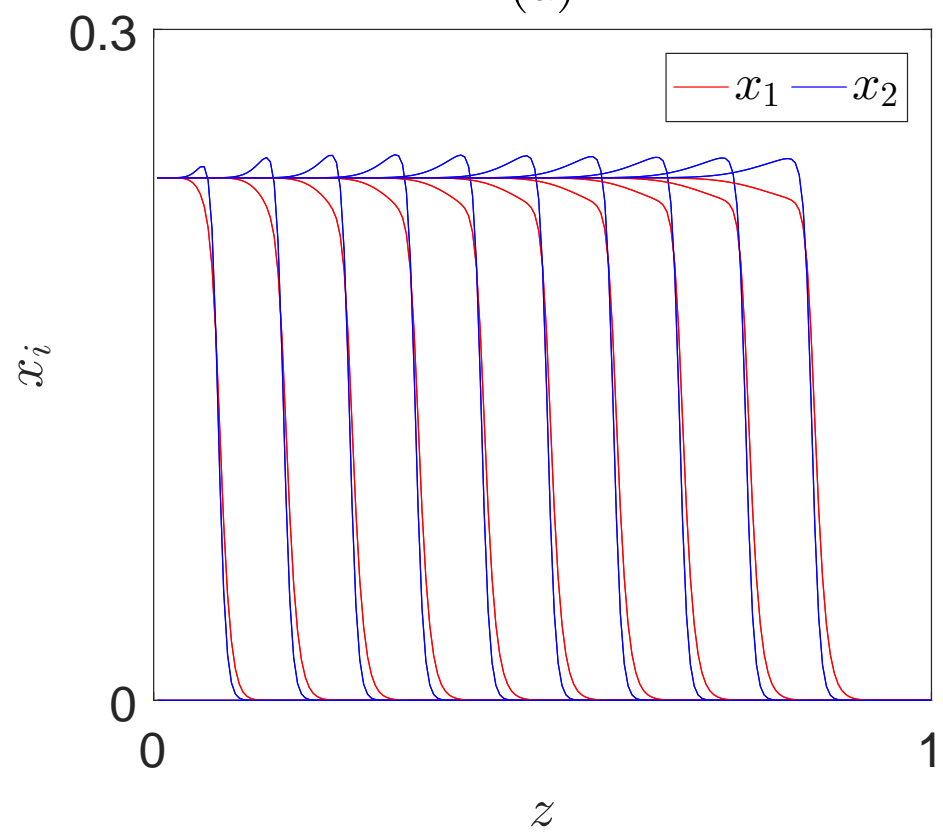

(b)

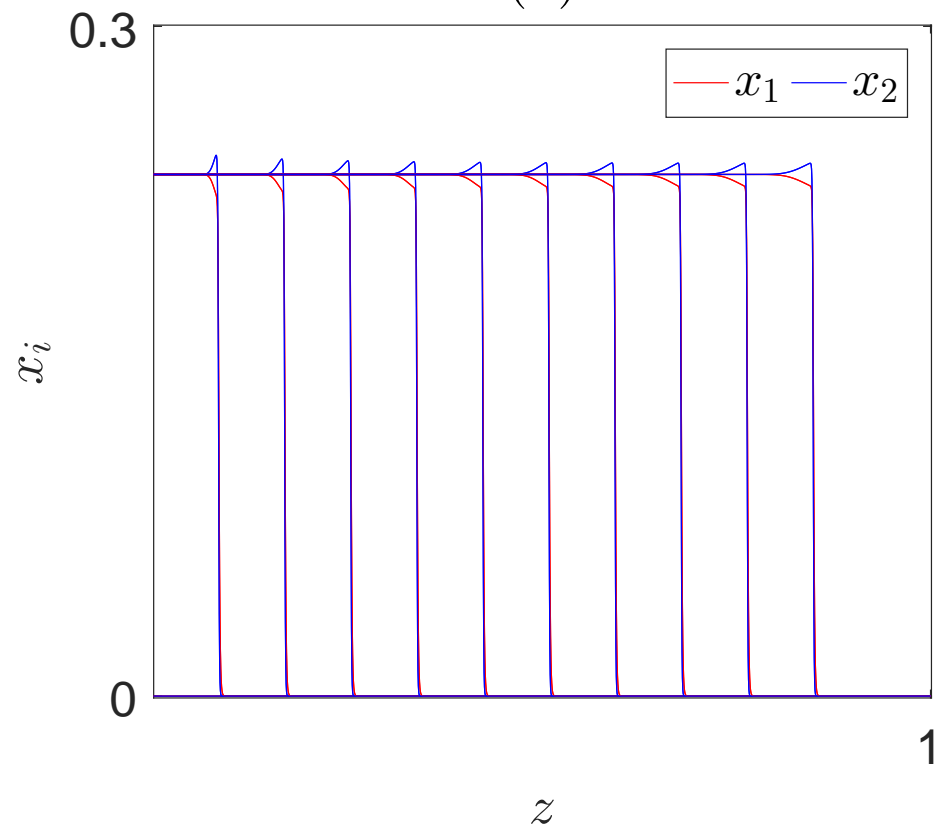

29

Figure 5: Numerical simulation with (a) 200 and (b) 2000 grid points for a feed on the selectivity reversal at $F=[7 / 30,7 / 30]^{T}$ corresponding to Fig. 4 
(a)

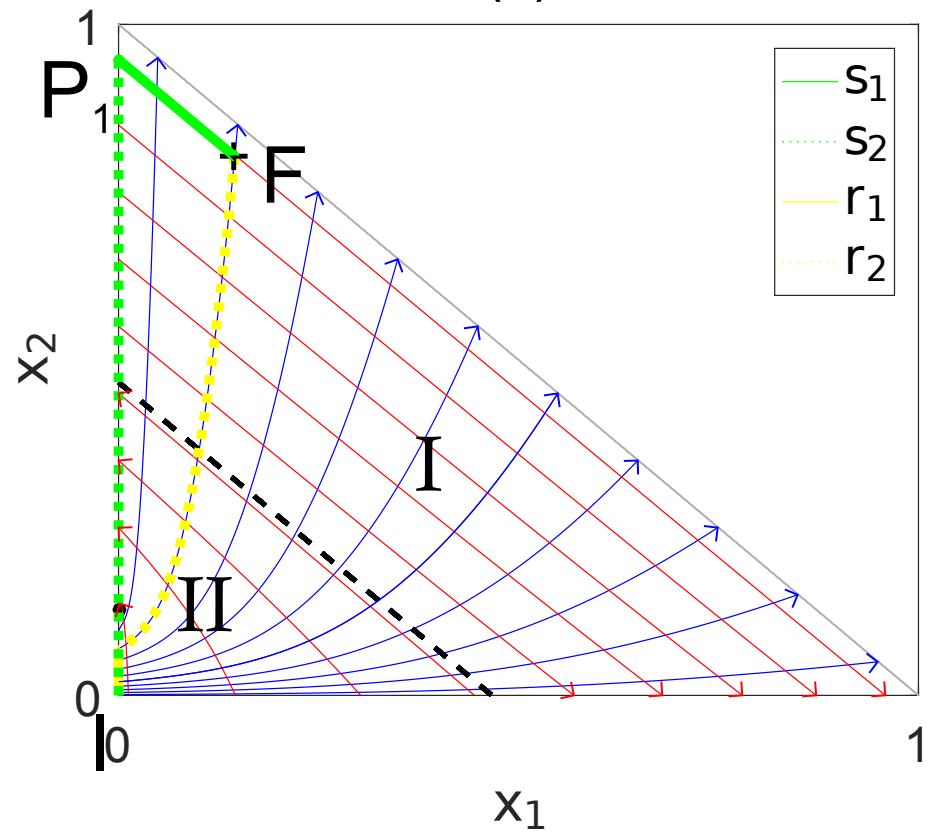

(b)

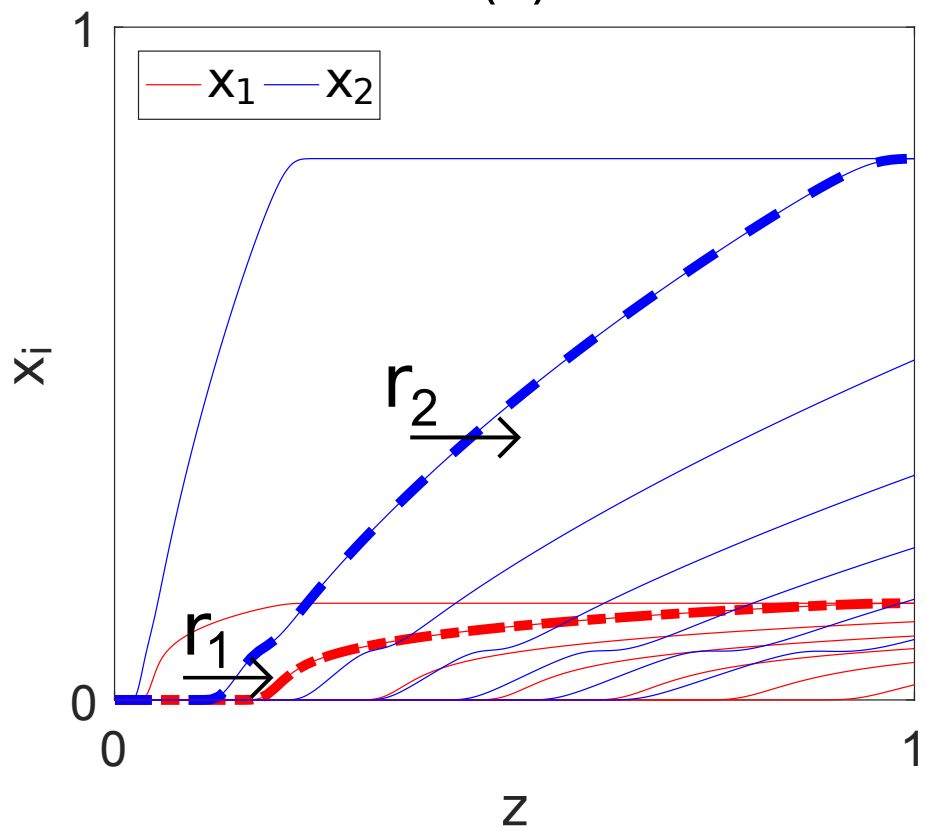

Figure 6: (a) Chromatographic cycle in the hodograph space for $F=[0.1437,0.8046]^{T}$ in I. (b) Corresponding spatial regeneration profiles $x_{i}(z)$ at different time points. 

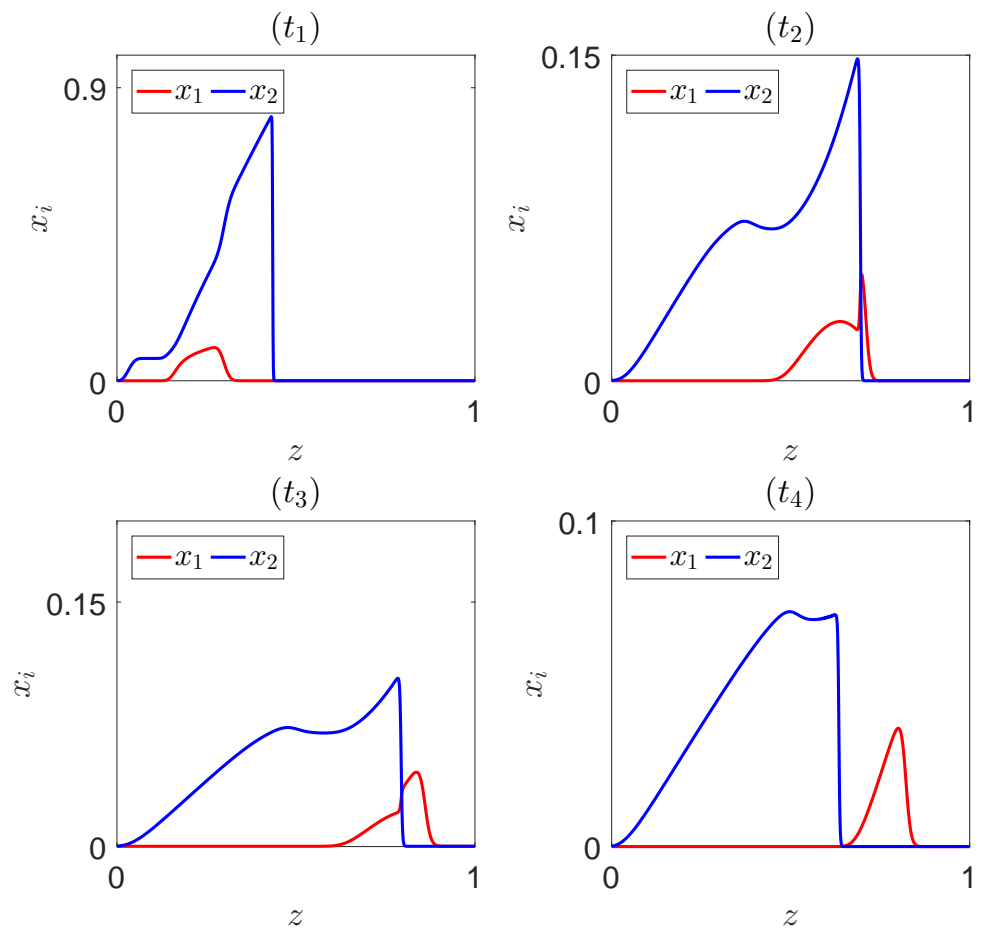

Figure 7: Spatial profiles at different time points for a pulse injection with plateau at $F=$ $[0.1437,0.8046]^{T}$ in I (see also Fig. 6a). 
(a)

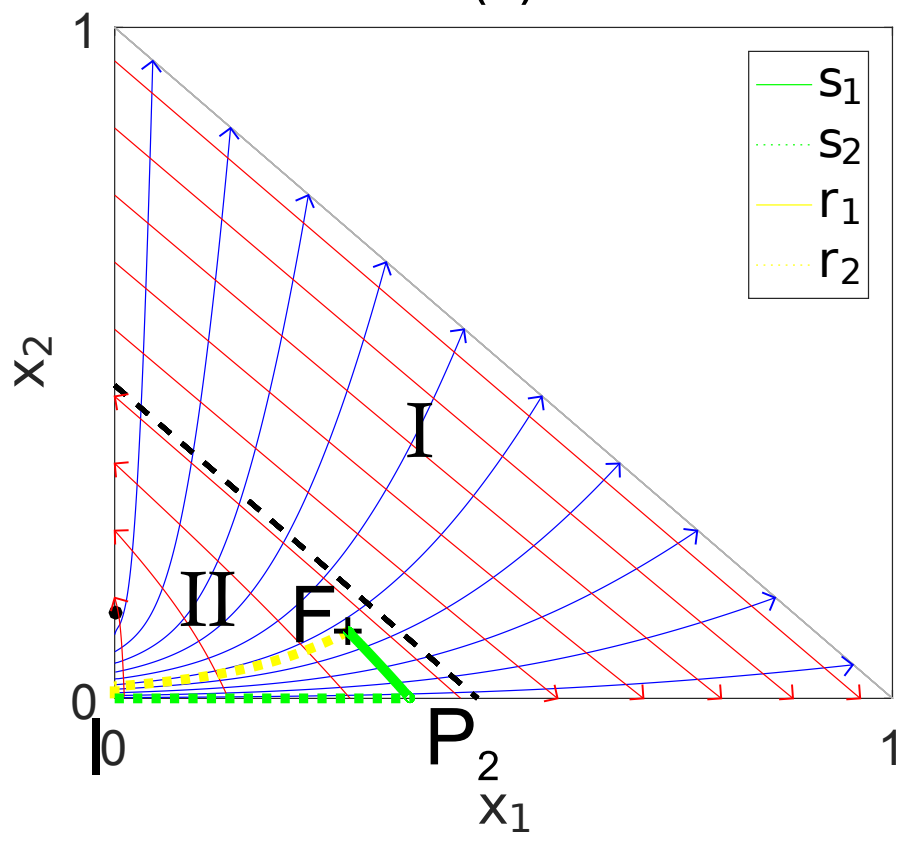

(b)

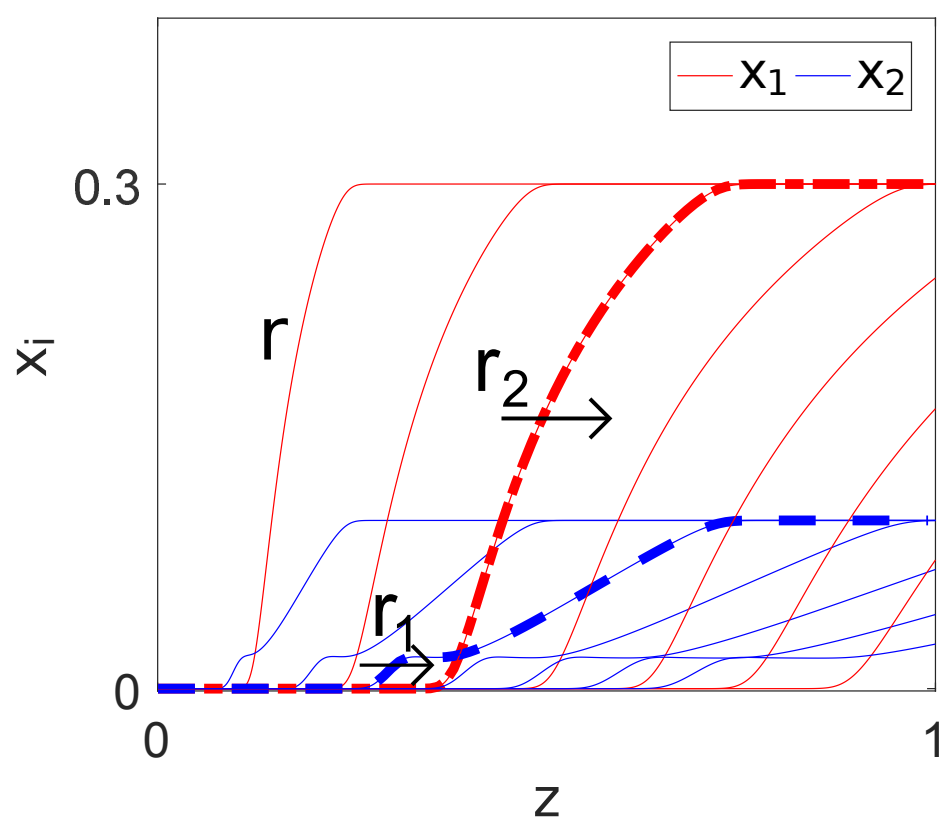

Figure 8: (a) Chromatographic cycle in the hodograph space for $F=[0.3,0.1]^{T}$ in II. (b) Corresponding spatial regeneration profiles $x_{i}(z)$ at different time points. 

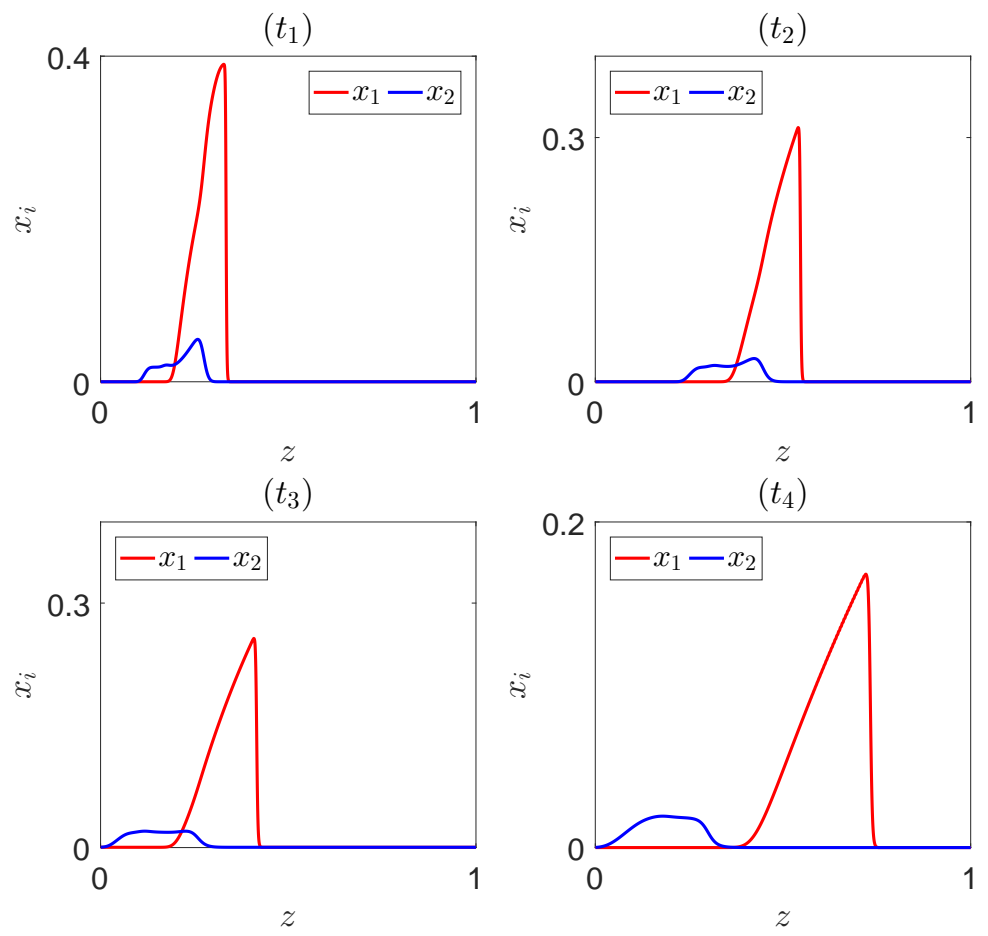

Figure 9: Spatial profiles at different time points for a pulse injection with plateau at $F=$ $[0.3,0.1]^{T}$ in II (see also Fig. 8a). 
(a)

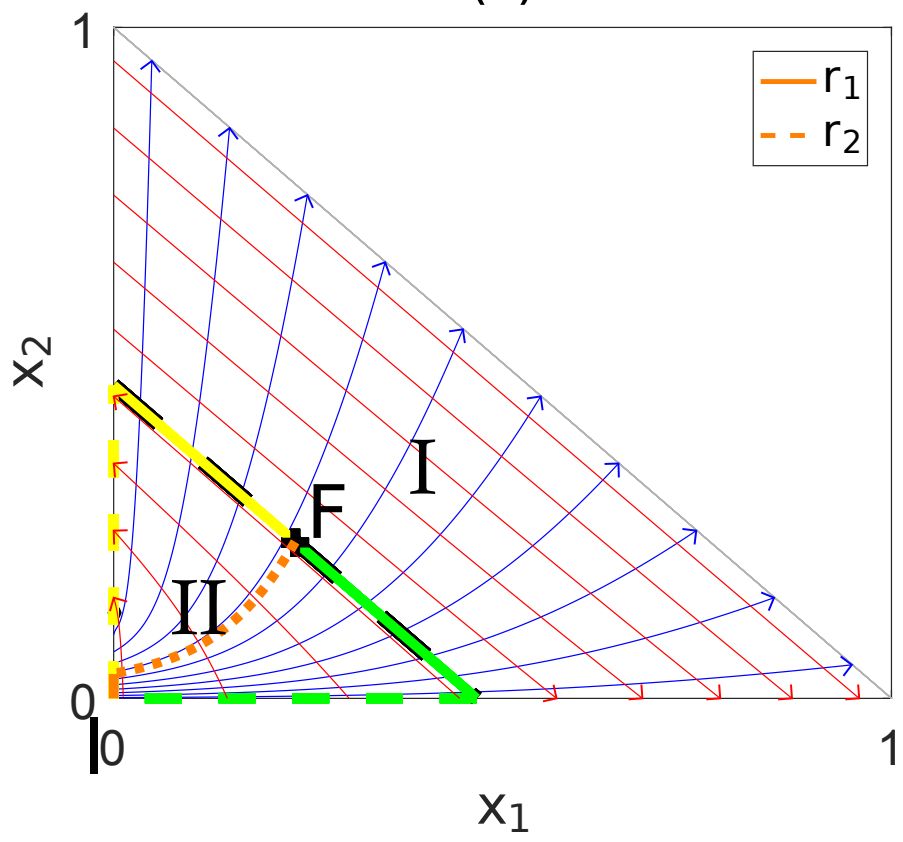

(b)

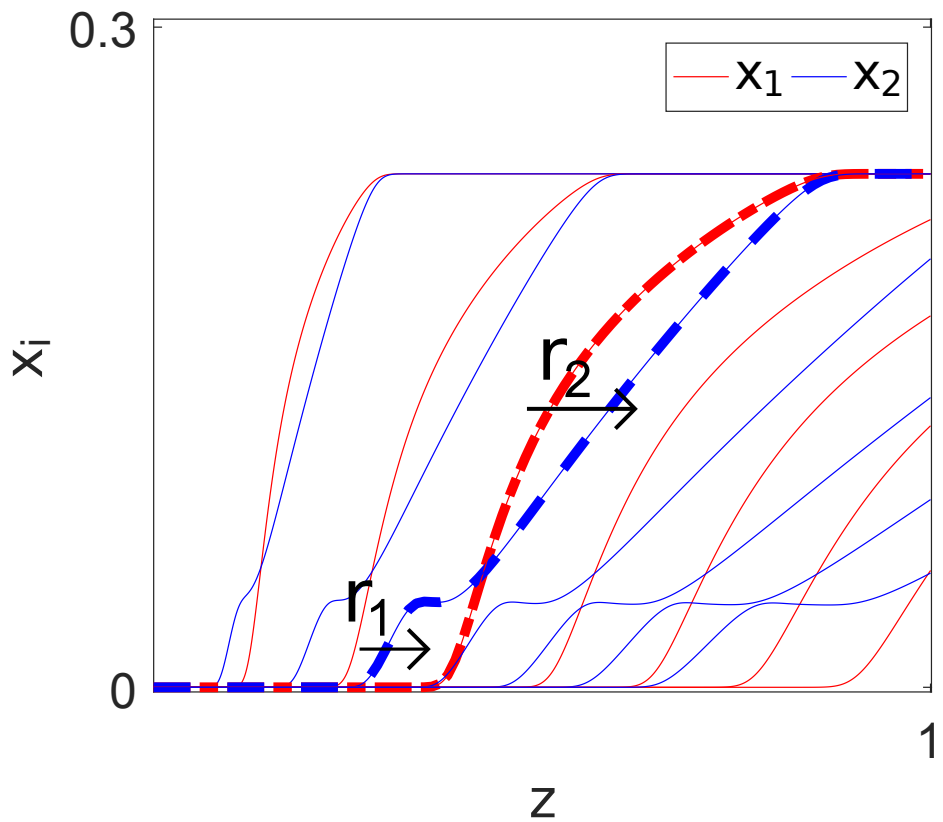

34

Figure 10: (a) Two possible chromatographic cycles in the hodograph space for $F=$ $[7 / 30,7 / 30]^{T}$ on the selectivity reversal. (b) Corresponding spatial regeneration profiles $x_{i}(z)$ at different time points. 
(a)

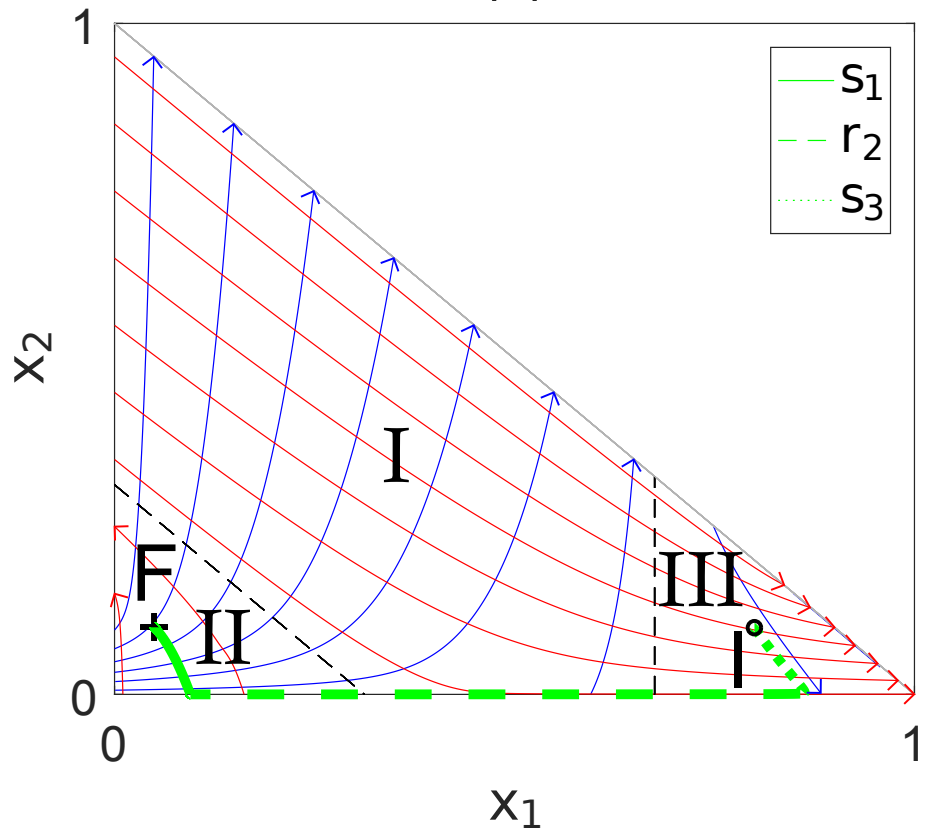

(b)

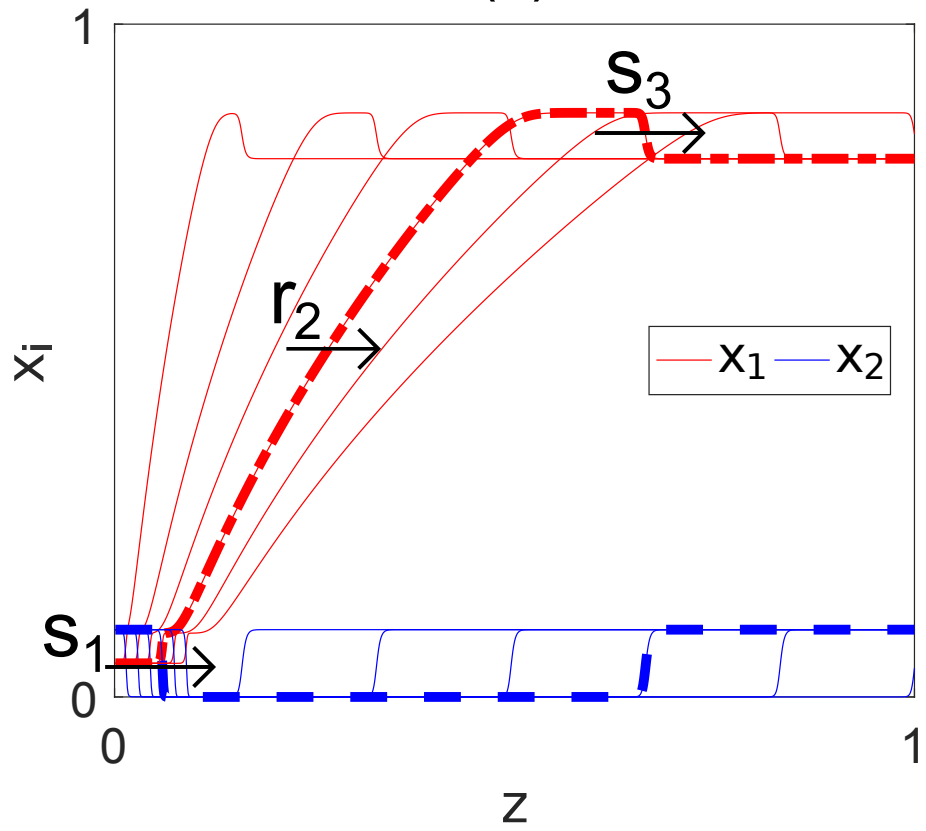

Figure 11: (a) Hodograph of the second example system with two selectivity reversals including a path from $F=[0.05,0.1]^{T}$ to $I=[0.8,0.1]^{T}$. (b) Corresponding spatial profiles $x_{i}(z)$ at different time points. 